\title{
Damped Jacobi Methods Based on Two Different Matrices for Signal Detection in Massive MIMO Uplink
}

\author{
Aounallah Naceur (D), \\ Department of Electronic and Telecommunications, Faculty of new information technologies and \\ communication, Kasdi Merbah University, Ouargla 30000, Algeria \\ aounallah.na@univ-ouargla.dz
}

\begin{abstract}
For massive multiple-input multiple-output (m-MIMO) uplink, the performances of the linear minimum mean-square error (MMSE) detector are considered near optimal, and they occupy benchmark place for most linear iterative detectors. However, the MMSE algorithm is known by its load computational complexity due to the implication of large-scale matrix inversions, and in other hand, iterative methods are often preferred in signal detection because of its low complexity. In this paper, we propose a New Damped Jacobi (NDJ) detector in order to improve the performance of the classical Jacobi linear algorithm. Starting from the classical Jacobi technique to our new proposal, we go through the development of two variants; one uses a damping factor and the other uses a stair-matrix. However, the NDJ incorporates a damping factor in its construction and basing also on stair matrix instead of diagonal matrix. The performances in terms of convergence and low complexity of each Jacobi variant studied in this paper are analyzed. Finally, some simulation examples are given to illustrate the advantages of the new proposed algorithm.
\end{abstract}

Index Terms - Massive MIMO, iterative Jacobi method, diagonal matrix, stair matrix, damping factor.

\section{INTRODUCTION}

A communication system structured on a massive antenna array, whose purpose is to serve simultaneously multiple users, is commonly called a massive MIMO system. This last represents one of the key and promising technologies for 5G mobile communications, in fact, it has the capability to combat the severe fading of mm-wave signals and to eliminate interference in multi-layer and denser networks, and it is also able to ensure wireless links [1]. In addition, this system takes care to achieve some challenges among which the increasing complexity of the detection task especially when the number of used antennas is large.

Signal detection in massive MIMO uplink is a core technology for the future 5G wireless communications, furthermore, detecting efficiently and accurately signals in such new system is of vital importance [2]. In literature, the set of algorithms used for massive MIMO signal detection can be classified either into linear detection algorithm or nonlinear detection algorithm according to different calculation methods [3]. Although the linear algorithms are less complex compared to the nonlinear ones, however, their main difficulty goes back to the calculation of the inverse matrix of a Brazilian Microwave and Optoelectronics Society-SBMO received 22 May 2020; for review 31 May 2020 ; accepted 10 Dec 2020 
higher rank matrix created mostly from a massive MIMO system of very large scale.

In recent years, many researchers in the field of mobile communications and massive MIMO technology have achieved abundant works concerning system uplink detection. The general purpose of their researches was to achieve good tradeoff between the performance and the computational complexity of the system. Additionally, various low-complexity detection schemes have been developed in order to reach near-optimal performance [4]-[6].

Among the well know iterative linear methods which have been developed to avoid or to approximate channel matrix inversion there are Jacobi method [7], Gauss-Seidel method [8], successive over-relaxation (SOR) method [9], Newton iterations method [10], Richardson method [11], and Neumann series [12]. The low-complexity of Jacobi iterative method leads some authors to work on improving its performance in terms of convergence rate. The refinement of Jacobi iterative method proposed in [13] has offered a new low-complexity MMSE detector, in fact, this refinement has been given by the use of band matrix to accelerate the convergence rate and guarantee the nearoptimal BER performance. In [14], a Jacobi method-based scheme has been established by using an initial estimation provided by steepest descent technique. In the same context, authors of [15] have proposed a novel joint conjugate gradient and Jacobi iteration method to speed up the convergence rate with lower implementation complexity and latency. A decision-aided Jacobi (DA-Jacobi) iteration has been proposed in [16], this proposition can extensively improve the convergence speed as compared to the traditional Jacobi, and at the same time, may result in lower computational complexity. By providing a damping factor [17], a damped Jacobi method has been appeared lately to reduce the computational complexity of the classical MMSE detector, and to ensure the fast convergence thanks to the multiplication of the initial solution by a reasonable damping factor.

Here it is worth pointing out that most proposals in existing literature extensively use the diagonal matrix in their development to solve linear equations. Furthermore, the application of stair matrix in massive MIMO systems has been recently taken into account in [18] when the authors have addressed two fundamental issues in the development of their iterative method using stair matrix; 1- the probability that the convergence conditions are satisfied for saying whether the stair matrix is applicable or not in massive MIMO, and 2- the convergence rate which reveals the advantages over the use of diagonal matrix.

The rest of this paper is organized as follows. Section II introduces the Massive-MIMO system model. Section III describes briefly and shows the form of diagonal and stair matrices. Section IV reviews the conventional Jacobi method, then presents the Damped Jacobi version, after that generalizes the Jacobi linear detector based on stair matrix, then explains the principle of the New Damped Jacobi (NDJ) linear detector using stair matrix, and finally discusses the computation complexity analysis. The performances of the discussed approaches are validated by means of numerical and simulation results in section V. Finally, the conclusion is drawn in section VI. 


\section{SYSTEM MODEL}

We consider the uplink of a massive MIMO system as shown in Fig.1, in such system, $U$ singleantenna users communicate with the base station (BS) which is equipped with $N$ antennas, where $N \gg U$. The modulated data symbols $(s)$ are transmitted through the massive MIMO channel described by $H \in C_{N \times U}$. Each element in $H$ is independently generated by the complex Gaussian random variable with zero mean and unit variance.

The received signal vector $y \in C_{N \times 1}$ at base station can be expressed as:

$$
y=H s+n
$$

with $n \in C_{N \times 1}$ is the zero-mean complex Gaussian distributed noise vector with variance $\sigma_{n}^{2}$.

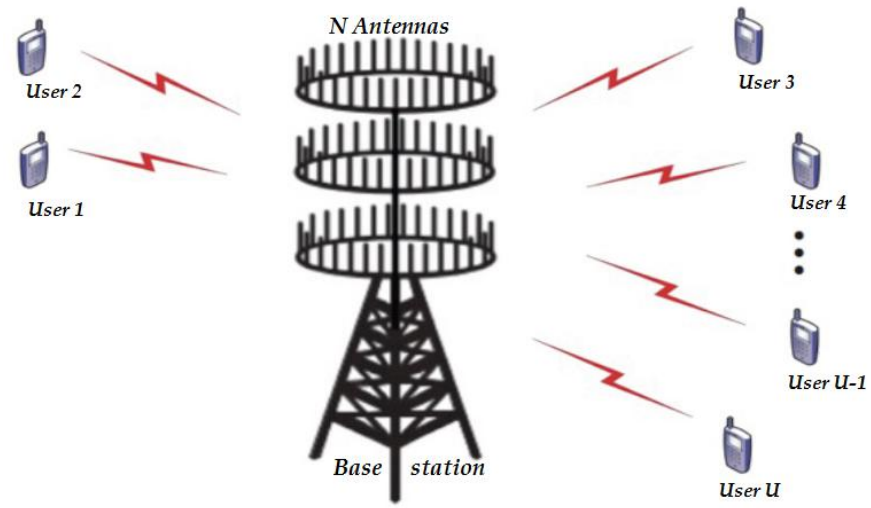

Fig. 1. Massive MIMO model with $\mathrm{N}$ antennas at BS to serve $\mathrm{U}$ users.

The transmitted signal can be recovered by the MMSE detector and expressed as:

$$
\hat{s}=\left(H^{H} H+\sigma_{n}^{2} I\right)^{-1} H^{H} y=A^{-1} y_{M F}
$$

Where $A=G+\sigma_{n}^{2} I$ denotes the MMSE filtering matrix, $I$ is the identity matrix, $G=H^{H} H$ is the Gram matrix, and $y_{M F}=H^{H} y$ represents the matched-filter output of $y$.

\section{FROM DIAGONAL MATRIX TO STAIR MATRIX}

In theory, a matrix $D$ is called diagonal matrix if its elements outside the main diagonal are all zeros [19], for instance, a $6 \times 6$ diagonal matrix can be given as:

$$
D=\left[\begin{array}{llllll}
\mathrm{x} & 0 & 0 & 0 & 0 & 0 \\
0 & \mathrm{x} & 0 & 0 & 0 & 0 \\
0 & 0 & \mathrm{x} & 0 & 0 & 0 \\
0 & 0 & 0 & \mathrm{x} & 0 & 0 \\
0 & 0 & 0 & 0 & \mathrm{x} & 0 \\
0 & 0 & 0 & 0 & 0 & \mathrm{x}
\end{array}\right]
$$


A tridiagonal matrix $T$ is a matrix whose all the elements on the main diagonal, super diagonal (above main diagonal) and sub-diagonal (below main diagonal) are non-zeros. All the rest of the elements are zeros, for example, a $6 \times 6$ tridiagonal matrix takes the following form:

$$
T=\left[\begin{array}{llllll}
\mathrm{x} & \mathrm{x} & 0 & 0 & 0 & 0 \\
\mathrm{x} & \mathrm{x} & \mathrm{x} & 0 & 0 & 0 \\
0 & \mathrm{x} & \mathrm{x} & \mathrm{x} & 0 & 0 \\
0 & 0 & \mathrm{x} & \mathrm{x} & \mathrm{x} & 0 \\
0 & 0 & 0 & \mathrm{x} & \mathrm{x} & \mathrm{x} \\
0 & 0 & 0 & 0 & \mathrm{x} & \mathrm{x}
\end{array}\right]
$$

A stair matrix is a special tridiagonal matrix that satisfies one of the following conditions:

1) $T_{(m, m-1)}=0, T_{(m, m+1)}=0$, where $m=2 k-1, k=1,2, \ldots,(N+1) / 2$. That means, the nondiagonal elements in the odd rows of tridiagonal matrix are zeros.

2) $T_{(m, m-1)}=0, T_{(m, m+1)}=0$, where $m=2 k, k=1,2, \ldots, N / 2$. And that means, the nondiagonal elements in the even rows of tridiagonal matrix are zeros.

According to the two above conditions, we can define a stair matrix $S$ as a tridiagonal matrix where the off-diagonal entries on either the odd or the even row are zeros [18]. For example, a $6 \times 6$ stair matrix can have one of the two following forms corresponding to the first and second previous conditions, respectively:

$$
S 1=\left[\begin{array}{cccccc}
\mathrm{x} & 0 & 0 & 0 & 0 & 0 \\
\mathrm{x} & \mathrm{x} & \mathrm{x} & 0 & 0 & 0 \\
0 & 0 & \mathrm{x} & 0 & 0 & 0 \\
0 & 0 & \mathrm{x} & \mathrm{x} & \mathrm{x} & 0 \\
0 & 0 & 0 & 0 & \mathrm{x} & 0 \\
0 & 0 & 0 & 0 & \mathrm{x} & \mathrm{x}
\end{array}\right] \quad \text { or } \quad S 2=\left[\begin{array}{cccccc}
\mathrm{x} & \mathrm{x} & 0 & 0 & 0 & 0 \\
0 & \mathrm{x} & 0 & 0 & 0 & 0 \\
0 & \mathrm{x} & \mathrm{x} & \mathrm{x} & 0 & 0 \\
0 & 0 & 0 & \mathrm{x} & 0 & 0 \\
0 & 0 & 0 & \mathrm{x} & \mathrm{x} & \mathrm{x} \\
0 & 0 & 0 & 0 & 0 & \mathrm{x}
\end{array}\right]
$$

The stair matrix $S=\operatorname{stair}\left(s_{m, m-1}, s_{m, m}, s_{m, m+1}\right)$ is nonsingular if and only if $s_{m m}, m=1,2, \ldots, N$ are nonsingular, furthermore, its inverse $S^{-1}$ is also a stair matrix given by:

$$
S^{-1}=D_{s}^{-1}\left(2 D_{s}-S\right) D_{s}^{-1}
$$

where $D_{s}=\operatorname{diag}\left(s_{11}, s_{22}, \ldots, s_{m m}\right)$ is diagonal matrix extracted from the stair matrix $S$.

Then, a stair linear system $S x=b$ can be easily solved by computing $S^{-1} b$.

\section{DETECTION ALGORITHMS}

For this section, we mainly focus on the description of a well known iterative method which has been proposed recently to solve the MMSE algorithm problem for signal detection in Massive MIMO systems; it's about the Jacobi method. From this last, we derive then different variants. 


\section{A. Conventional Jacobi method}

The iterative conventional Jacobi (CJ) method is one of the promising approaches that have been presented to avoid the direct calculation of matrix inversion. The basic idea of this method is to reexpress the MMSE estimation equation, then apply a matrix decomposition [7]. In other words, Jacobi iterative method can solve efficiently diagonally dominant systems of linear equation $A x=b$.

Rewriting equation (2) to:

$$
A \hat{s}=y_{M F}
$$

Decomposing the matrix $A$ into two parts: a diagonal-component matrix $D$ and a remainder matrix $R$ as $A=D+R$. The diagonal elements of $D$ are identical to those of $A$ and its offdiagonal elements are zeros, while the diagonal elements of $R$ are zeros and its off-diagonal elements are identical to those of $A$.

$$
D=\left[\begin{array}{cccc}
a_{11} & 0 & \cdots & 0 \\
0 & a_{22} & \cdots & 0 \\
\vdots & \vdots & \ddots & \vdots \\
0 & 0 & \cdots & a_{N N}
\end{array}\right]
$$

and

$$
R=\left[\begin{array}{cccc}
0 & a_{12} & \cdots & a_{1 N} \\
a_{21} & 0 & \cdots & a_{2 N} \\
\vdots & \vdots & \ddots & \vdots \\
a_{N 1} & a_{N 2} & \cdots & 0
\end{array}\right]
$$

By employing the Jacobi iterative method in the detection, the estimated-transmitted signal vector $\hat{s}$ can be obtained iteratively as follows [16]:

$$
\begin{aligned}
\hat{s}_{i+1} & =D^{-1}\left[R \hat{s}_{i}+y_{M F}\right] \\
& =D^{-1}\left[(D-A) \hat{s}_{i}+y_{M F}\right]
\end{aligned}
$$

where $\hat{s}_{i}$ and $\hat{s}_{i+1}$ are the approximations of $\hat{s}$ in the $i^{\text {th }}$ and $(i+1)^{\text {th }}$ iterations, respectively.

Since the convergence rate of the iteration is highly based on the initial setting, we consider the initial solution $s_{0}$ as:

$$
s_{0}=D^{-1} y_{M F}
$$

A sufficient but not necessary condition that guarantees convergence for the Jacobi iteration is diagonally dominance. In this case and according to the theorem 8.3.7 of [20], the Jacobi method converges if and only if the spectral radius $\rho\left(B_{C J}\right)$ of its iteration matrix is less than one. This is expressed mathematically as:

$$
\rho\left(B_{C J}\right)=\left|\lambda_{\max }\left(B_{C J}\right)\right|<1
$$


where $B_{C J}=-D^{-1}(A-D)=I-D^{-1} A$ is the iteration matrix of conventional Jacobi, and $\lambda_{\max }\left(B_{C J}\right)$ denotes largest eigenvalue of $B_{C J}$.

\section{B. Damped Jacobi method}

Based on the ordinary Jacobi iteration method, the damped version (DJ) is a simple way which is used to improve the system detection performance in terms of convergence speed. In fact, the estimations correction $\left(D^{-1}\left(A \hat{s}_{i}-y_{M F}\right)\right)$ at each iteration is damped by real parameter $\omega$, thus, the damped Jacobi iterative method can be expressed as:

$$
\hat{s}_{i+1}=\hat{s}_{i}-\omega D^{-1}\left(A \hat{s}_{i}-y_{M F}\right)
$$

From expression (13), it can be shown that:

$$
\begin{gathered}
\hat{s}_{i+1}=\hat{s}_{i}+\left(\omega \hat{s}_{i}-\omega \hat{s}_{i}\right)-\omega D^{-1}\left(A \hat{s}_{i}-y_{M F}\right) \\
=\hat{s}_{i}(1-\omega)-\omega D^{-1}\left(A \hat{s}_{i}-y_{M F}-D \hat{s}_{i}\right) \\
=\hat{s}_{i}(1-\omega)+\omega D^{-1}\left((D-A) \hat{s}_{i}+y_{M F}\right) \\
=(1-\omega) \hat{s}_{i}+\omega D^{-1}\left((D-A) \hat{s}_{i}+y_{M F}\right) \\
=(1-\omega) \hat{s}_{i}+\omega D^{-1}\left(y_{M F}-R \hat{s}_{i}\right)
\end{gathered}
$$

For the signal detection using damped Jacobi method, we can use an initial solution given as follows:

$$
s_{0}=D^{-1} y_{M F}
$$

By generalizing the convergence condition of the Jacobi method, we obtain that the convergence for its damped version is ensured by the following constraint:

$$
\rho\left(B_{D J}\right)=\left|\lambda_{\max }\left(B_{D J}\right)\right|<1
$$

where $\rho\left(B_{D J}\right)$ denotes the spectral radius of the iteration matrix of the damped Jacobi which is $B_{D J}=(1-\omega) I-\omega D^{-1}(A-D)=I-\omega D^{-1} A$, and $\lambda_{\max }\left(B_{D J}\right)$ denotes the biggest eigenvalue of $B_{D J}$.

In addition, choosing a good damping factor value plays an important role in the convergence of the damped Jacobi method. This method convergences regularly when $\omega$ satisfies $0 \leq \omega \leq 2 / \lambda_{\max }$.

\section{Jacobi based on stair matrix}

Jacobi based on stair matrix (JS) algorithm is considered as a modified Jacobi approach that uses a stair matrix in the MMSE filtering matrix decomposition instead of a diagonal matrix. Such iterative method based on the stair matrix class can be easily implemented for parallel computation [19]. In this particular case, the iterative algorithm structure is formulated as:

$$
\hat{s}_{i+1}=S^{-1}\left[R_{s} \hat{s}_{i}+y_{M F}\right]
$$

Brazilian Microwave and Optoelectronics Society-SBMO received 22 May 2020; for review 31 May 2020; accepted 10 Dec 2020 


$$
\begin{aligned}
& =S^{-1}\left[(S-A) \hat{s}_{i}+y_{M F}\right] \\
& =\hat{s}_{i}-S^{-1} A \hat{s}_{i}+S^{-1} y_{M F}
\end{aligned}
$$

where $\hat{S}_{i}$ denotes the $i^{\text {th }}$ estimation, $S$ is the stair matrix, and $A$ remains always the MMSE filtering matrix.

For this modified Jacobi algorithm that uses a stair matrix, the initial estimation is established as:

$$
s_{0}=S^{-1} y_{M F}
$$

Basing on the same convergence conditions of conventional Jacobi method, we come back to find that the sufficient condition which requires a Jacobi-stair to converge is as follows:

$$
\rho\left(B_{J S}\right)=\left|\lambda_{\max }\left(B_{J S}\right)\right|<1
$$

For the Jacobi-stair method, the iteration matrix is calculated using the stair matrix $S$ and it is given as follows:

$$
B_{J S}=-S^{-1}(A-S)=I_{u}-S^{-1} A
$$

\section{Proposed method}

The idea of our new proposed algorithm is to adopt the use of stair matrix in a damped Jacobi version. Thus, this proposition which is named new damped Jacobi (NDJ) detector combines both advantages of the Jacobi based on stair matrix instead of diagonal matrix and the damped Jacobi which is based on a damping parameter.

In layman terms, the proposed iterative algorithm expression can be easily deduced from the combination of the equations (13) and (17) as follows:

$$
\begin{aligned}
\hat{s}_{i+1} & =\hat{s}_{i}-\omega S^{-1}\left(A \hat{s}_{i}-y_{M F}\right) \\
& =(1-\omega) \hat{s}_{i}+\omega S^{-1}\left(y_{M F}-R_{s} \hat{s}_{i}\right)
\end{aligned}
$$

where $R_{s}=A-S$, and $\omega$ being the damping factor.

The initial estimation of the proposed algorithm (NDJ or DJS) can be expressed as:

$$
s_{0}=S^{-1} y_{M F}
$$

We summarize the complete steps of the proposed method output detection in algorithm I.

\section{Algorithm I: Damped Jacobi detector using stair matrix}

Input: $H, y, \sigma_{n}^{2}, N, U, i$, and $\omega$.

Output: $\hat{s}$ : the estimated value of $s$

\section{Initialization}

1: $G=H^{H} H, A=G+\sigma_{n}^{2} I, y_{M F}=H^{H} y$,

2: $S=\operatorname{stair}\left(A_{n, n-1}, A_{n, n}, A_{n, n+1}\right)$

3: $R_{s}=A-S$

4: $s(0)=S^{-1} y_{M F} ;$ Initial estimation 


\section{Iteration}

For $k=1, \ldots, i$ do

$$
s(k+1)=(1-\omega) s(k)+\omega S^{-1}\left(y_{M F}-R_{s} s(k)\right)
$$

\section{end for}

Return: $\hat{s}=s(k+1)$

To have a guarantee on the convergence of damped-Jacobi-stair variant, it is necessary that the spectral radius of its iteration matrix, which is constituted by both a damping parameter and a stair matrix, fulfills the following condition:

$$
\rho\left(B_{D J S}\right)=\left|\lambda_{\max }\left(B_{D J S}\right)\right|<1
$$

with:

$$
B_{D J S}=(1-\omega) I-\omega D^{-1}(A-S)=I-\omega S^{-1} A
$$

From (24) the eigen-values of the iteration matrix of damped Jacobi based on stair matrix $B_{D J S}$ are included in the following range:

$$
1-\omega \lambda_{\min }\left(S^{-1} A\right) \leq \lambda_{n}\left(B_{D J S}\right) \leq 1-\omega \lambda_{\max }\left(S^{-1} A\right)
$$

where $\lambda_{n}\left(B_{D J S}\right)$ is the $n^{\text {th }}$ eigen-value of $B_{D J S}$.

From (23) and (25) we can deduce that two conditions are required for the convergence of DampedJacobi-stair variant:

$$
\begin{array}{ll}
1-\omega \lambda_{\min }<1 & \Rightarrow \omega>0 \\
1-\omega \lambda_{\min }>-1 & \Rightarrow \omega<2 / \lambda_{\max }
\end{array}
$$

The two previous expressions can be reformulated into one as follows:

$$
0 \leq \omega \leq 2 / \lambda_{\max }
$$

The convergence analysis of our four studied algorithms leads us to classify them according to the speed convergence rate as follows; damped Jacobi based on stair matrix, damped Jacobi, Jacobi based on stair matrix, and then conventional Jacobi. This ranking results from the comparison between the spectral radius of iteration matrices which shows the following:

$$
\rho\left(B_{D J S}\right)<\rho\left(B_{D J}\right)<\rho\left(B_{J S}\right)<\rho\left(B_{C J}\right)
$$

So, the smaller spectral radius is, the faster the iteration method converges.

\section{E. Computational complexity}

One of the essential concepts in evaluating the performance of massive MIMO detection algorithms is the computational complexity. To evaluate the computational complexity of our studied algorithms we need to analyze it in terms of the number of real number multiplications-divisions. For theoretical analysis, the overall computational complexity is split into two parts which are the initialization step and the iterations process. For our proposed method where its important steps are pointed out above 
in Algorithm I, in the initialization part, the computation of $A, y_{M F}$ and $S^{-1}$, needs $2 N U^{2}, 4 N U$ and ( $4 U-3)$ respectively. In addition, the initial estimation computation requires $2 U+2 \times \frac{U+1}{2}+(8+2) \times \frac{U-1}{2}=8 U-4$. Thus, the total initialization computation is $2 N U^{2}+4 N U+12 U-7$. On another side, in the iteration part, the calculation of $(1-\omega) s(k)$ requires $\quad U+6 U-4=7 U-4 \quad$ and $\quad \omega S^{-1}\left((S-A) s(k)+y_{M F}\right) \quad$ requires $2 U \times(U-1)+4(U-1) \times \frac{U+1}{2}+4(U-3) \times \frac{U-1}{2}=6 U^{2}-10 U+4$. Thus, the total computation of the iteration part is $i\left(6 U^{2}-3 U\right)$.

A comparison of computational complexity between the different studied Jacobi variants that can be used for $N \times U$ massif MIMO detector is shown in Table I.

TABLE I. COMPUTATIONAL COMPLEXITY COMPARISON

\begin{tabular}{ccc}
\hline Methods & Initialization step & Iteration process \\
\hline Conventional Jacobi (CJ) & $2 N U^{2}+4 N U$ & $i\left(4 U^{2}-2 U\right)$ \\
Damped Jacobi (DJ) & $2 U(N U+2 N+1)$ & $i\left(6 U^{2}-3 U\right)$ \\
Jacobi-Stair (JS) & $2 N U^{2}+4 N U+10 U-7$ & $i\left(4 U^{2}-2 U\right)$ \\
Damped Jacobi-Stair (DJS) & $2 N U^{2}+4 N U+12 U-7$ & $i\left(6 U^{2}-3 U\right)$ \\
\hline
\end{tabular}

From Table I, we can note that the damped Jacobi method as compared to the conventional Jacobi method adds $2 U$ and $\left(2 U^{2}-U\right)$ of complexity respectively in the initialization step and the iteration process, while the Jacobi based on stair matrix as compared to the conventional method adds $(10 U-7)$ of complexity only in its initialization step. It is also noteworthy that the proposed method brings a small increase in its complexity compared to that of the basic algorithm and which is determined as $(12 U-7)$ and $\left(2 U^{2}-U\right)$ in the initialization and the iteration parts, respectively. Further, the overall computational cost of all the previous analyzed methods is low and is in the same order which is $O\left(U^{2}\right)$. Hence the proposed method (NDJ) has a complexity which is quadratic with respect to the users number, that means one order of magnitude less than MMSE with direct matrix inversion. Therefore, the damped Jacobi based on stair matrix offers a new low-complexity algorithm for massive MIMO detection without complicated matrix inversion.

\section{SIMULATION RESULTS}

In this section, some simulations are designed to evaluate the performance of the proposed new damped Jacobi iteration algorithm. The Monte-Carlo simulation is followed and its results are provided on the BER performance to compare the proposed algorithm with the other studied Jacobi 
version detectors. The simulation environment is assumed to be uplink massive MIMO system equipped with 128 antennas at BS serving 32 single-antenna users and with 64-QAM modulation scheme.

The performance of the different studied Jacobi variants in massive MIMO systems are here evaluated in terms of BER curves and using the MMSE detector as the benchmark for comparison. The results are shown in figures 2, 3 and 4. As is observed from these figures, all modifications applied to the basic Jacobi algorithm provide improvements where each Jacobi version comes with its own enhancement degree which is different to that of another version. These modification versions can be classified according to the achieved improvement as follows; Damped-Jacobi-stair, DampedJacobi, then Jacobi-stair.

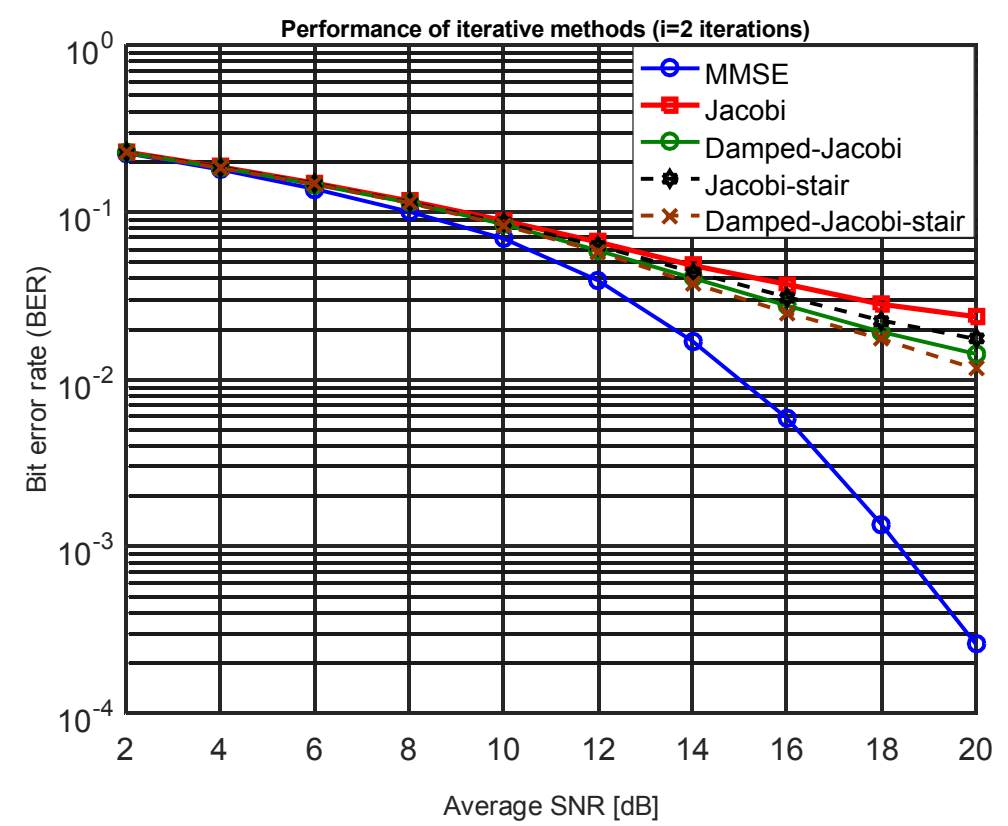

Fig. 2. BER performance comparison between different iterative methods for 2 iterations.

Comparing Fig. 2, Fig. 3 and Fig. 4, we can find that with an increasing number of iterations the BER performances of all considered iterative detectors improve where the BER gains between these detectors becomes more measurable. It can be seen also from Fig. 4 for example where $i=4$ that a small BER difference between the damped Jacobi variants is achieved. In addition, compared with the exact MMSE algorithm and for relatively small iterations, a satisfying performance can be achieved generally by the damped algorithms and more particularly by our new damped Jacobi basing on stair matrix. It is worth noting that the damping factor value plays a significant role in the algorithm convergence, and in our simulations a damping parameter equal to 0.6 is selected. 


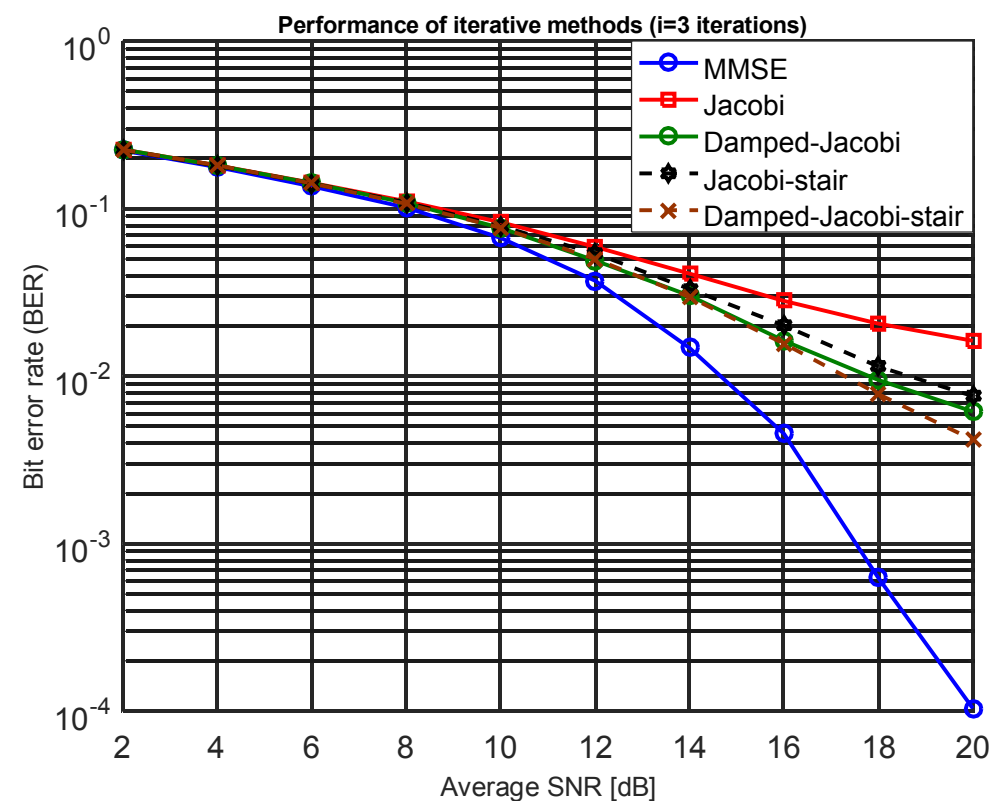

Fig. 3. BER performance comparison between different iterative methods for 3 iterations.

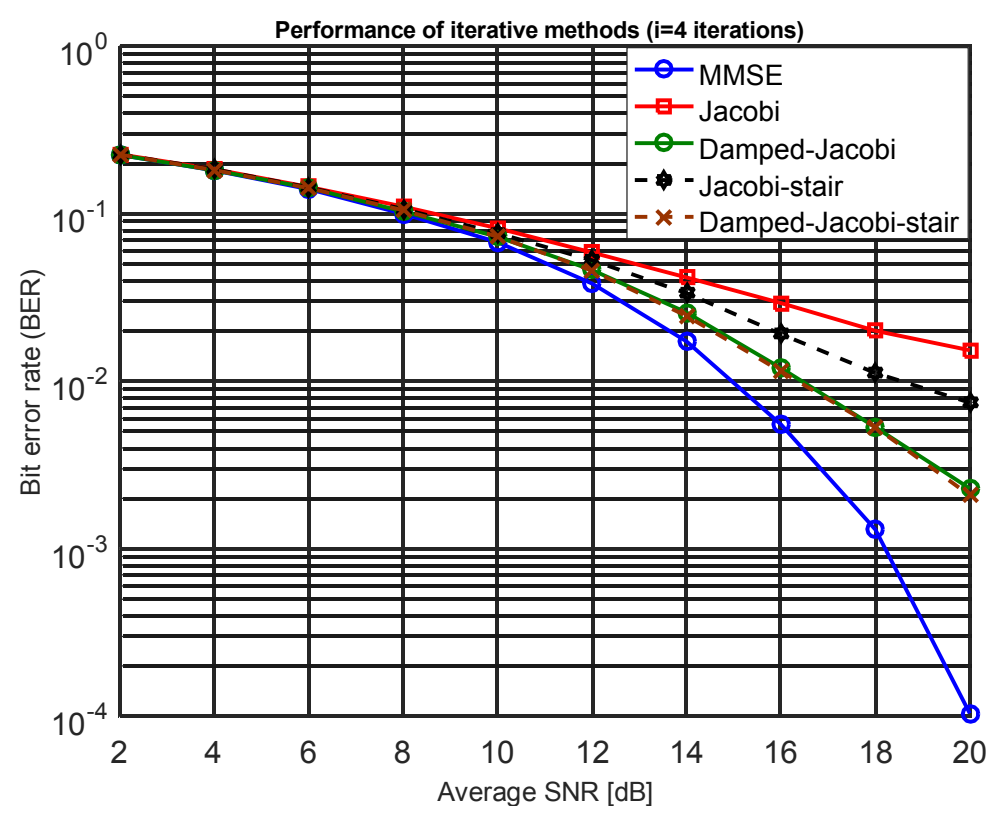

Fig. 4. BER performance comparison between different iterative methods for 4 iterations.

We also perform another simulation in order to show the influence of the increase in the number of BS antennas on the BER, and results are illustrated in Fig. 5. We test our proposed algorithm (NDJ) by considering in almost the same previous conditions a fixed number of users, $U=32$, but the number of BS antennas $N$ is varied from 64 to 140 , and a value of $\mathrm{SNR}=12 \mathrm{~dB}$ is adopted. It can be seen that, by increasing the number of BS antennas, the BER performance of both considered algorithms (MMSE and NDJ) improves. In addition, with a small number of iterations (i.e., $i=4$ ) and high number of antennas, the performance of the NDJ algorithm approximates the exact performance of the MMSE algorithm. Thus, higher $N$ is suggested to compensate the BER performance difference while retaining relatively small iteration value.

Brazilian Microwave and Optoelectronics Society-SBMO received 22 May 2020; for review 31 May 2020; accepted 10 Dec 2020 


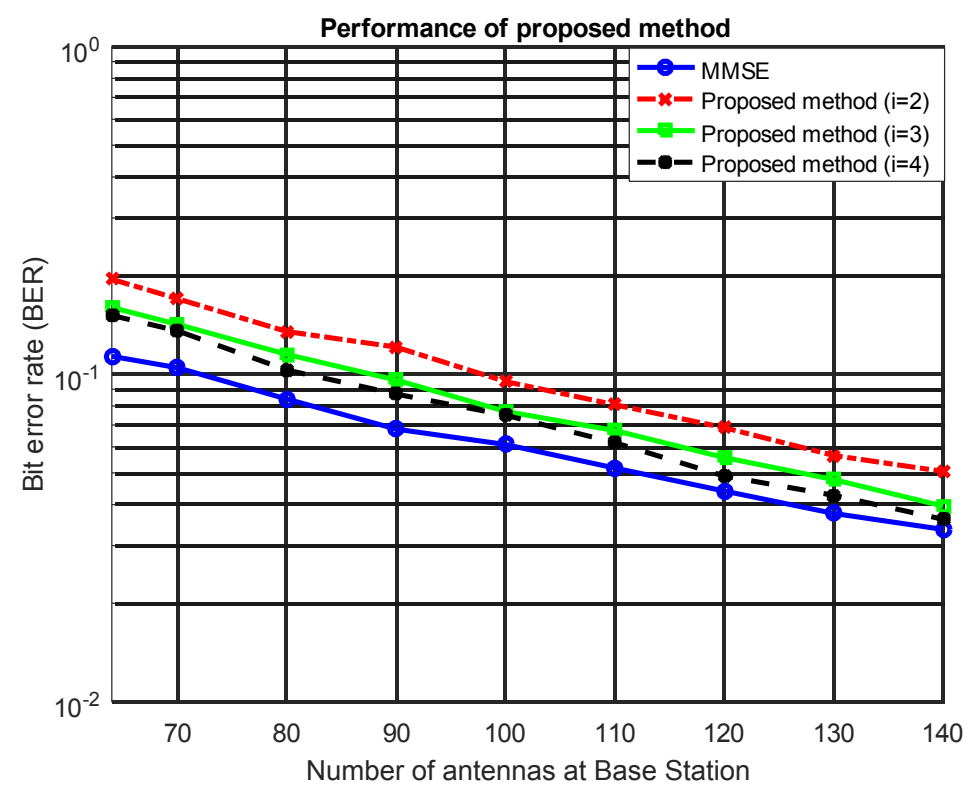

Fig. 5. BER performance comparison against the number of BS antennas.

\section{CONCLUSION}

The work presented in this paper introduces a new approach for massive MIMO signal detection based specially on the low complexity Jacobi algorithm. Moreover, using the matrices introduced in the present paper, we can develop and also create some other iterative scheme variants based on other conventional iterative detection techniques. The proposition of damped Jacobi scheme using stair matrix is a very new beneficial idea because it is able to reach a considerable compromise between speed convergence rate and low computational complexity. In fact, the new proposed algorithm which is called NDJ combines the both advantages of a damped approach and a simple decomposition technique basing on stair matrix.

\section{REFERENCES}

[1] L. Zhao, H. Zhao, K. Zheng, and W. Xiang, "Massive MIMO in 5G Networks: Selected Applications," Springer Briefs in Electrical and Computer Engineering, Switzerland, 2018.

[2] J. G. Andrews, S. Buzzi, C. Wan, et al. "What Will 5G Be?," IEEE J Sel Areas Commun, vol. 32, no. 6, pp. 1065-1082, 2014.

[3] S. P. Kim, J. C. Sanchez, Y. N. Rao, et al. "A Comparison of Optimal MIMO Linear and Nonlinear Models for BrainMachine Interfaces,” J Neural Eng, vol. 3, no. 2, pp. 145-161, 2006.

[4] M. Mahdavi, O. Edfors, V. Owall, and L. Liu, "A Low Complexity Massive MIMO Detection Scheme Using AngularDomain Processing," IEEE Global Conference on Signal and Information Processing (GlobalSIP), pp. 181-185, Nov 2018.

[5] S. Higuchi, and C. J. Ahn, "Reduced Complexity and Latency for a Massive MIMO System Using a Parallel Detection Algorithm," ICT Express, vol. 3, no. 3, pp. 119-123, Sept 2017.

[6] T. B. Nguyen, M. T. Le, and V. D. Ngo, "Signal Detection Based on Parallel Group Detection Algorithm for HighLoad Massive MIMO Systems," Wireless Communications and Mobile Computing, pp. 1-12, 2019.

[7] F. Jiang, C. Li, and Z. Gong, "A Low Complexity Soft-Output Data Detection Scheme Based on Jacobi Method for Massive MIMO Uplink Transmission," IEEE International Conference on Communications (ICC). Paris, France, May 2017.

[8] L. Dai, X. Gao, X. Su, S. Han, and Z. Wang, "Low-Complexity Soft-Output Signal Detection Based on Gauss-Seidel Method for Uplink Multiuser Large-Scale MIMO Systems," IEEE Trans. Veh. Technol., vol. 64, no.10, pp. 4839-4845, 2015.

[9] X. Gao, L. Dai, Y. Hu, Z. Wang, and Z. Wang, "Matrix Inversion-Less Signal Detection Using SOR Method for Uplink Large-Scale MIMO Systems,” IEEE Global Commun (GLOBECOM), pp. 3291-3295, Austin, TX, Dec 2014. 
[10] F. Jin, Q. Liu, H. Liu, and P. Wu, “A Low Complexity Signal Detection Scheme Based on Improved Newton Iteration for Massive MIMO Systems," IEEE Communications Letters, vol. 23, no. 4, pp. 748-751, 2019.

[11] X. Gao, L. Dai, C. Yuen, and Y. Zhang, "Low-Complexity MMSE Signal Detection Based on Richardson Method for Large-Scale MIMO Systems," IEEE 80th Vehicular Technology Conference, pp. 1-5, Sept 2014.

[12] M.Wu, B.Yin, A. Vosoughi, C. Studer, J.R. Cavallaro, and C. Dick, "Approximate Matrix Inversion for High Throughput Data Detection in The Large-Scale MIMO Uplink," IEEE ISCAS Conf, pp. 2155-2158, Beijing, China, May 2013.

[13] J. Minango, and A. C. Flores, "Low-complexity MMSE Detector Based on Refinement Jacobi Method for Massive MIMO Uplink," Physical Communication, vol. 26, pp. 128-133, 2018.

[14] X. Qin, Z. Yan, and G. He, "A Near-Optimal Detection Scheme Based on Joint Steepest Descent and Jacobi Method for Uplink Massive MIMO Systems," IEEE Commun. Lett., vol. 20, no. 2, pp. 276-279, 2016.

[15] W. Song, X. Chen, L. Wang, and X. Lu, "Joint Conjugate Gradient and Jacobi Iteration Based on Low Complexity Precoding for Massive MIMO Systems", IEEE/CIC International Conference on Communications in China (ICCC), pp. 1-5, Chengdu, 2016.

[16] L. Yinman, "Decision-aided Jacobi Iteration for Signal Detection in Massive MIMO Systems," Electronics Letters, vol. 53, no. 23, pp. 1552-1554, 2017.

[17] J. Minango, D. Altamirano, and C. de Almeida, "Low-complexity MMSE detector for massive MIMO systems based on damped Jacobi Method", IEEE 28th Int. Symposium on Personal, Indoor and Mobile Radio Commun (PIMRC), Montreal, Canada, Oct. 2017.

[18] F. Jiang, C. Li, Z. Gong, and R. Su, "Stair Matrix and its Applications to Massive MIMO Uplink Data Detection," IEEE Trans. Commun., vol. 66, no. 6, pp. 2437-2455, 2018.

[19] H. Lou, "Stair Matrices and Their Generalizations with Applications to Iterative Methods I: A Generalization of the Successive Over-relaxation Method," SIAM Journal on Numerical Analysis, vol. 37, no. 1, pp. 1-17, 2000.

[20] David S Watkins, "Fundamentals of matrix computations", third edition, John Wiley \& Sons, 2010. 